\title{
The Influence of Numerical Integration Accuracy and Sound Frequency on Computed Results of Acoustic Diffraction Problems with the Boundary Element Method
}

\author{
LiFeng \\ School of Automotive Engineering \\ GuangDong Polytechnic Normal University \\ Guangzhou, China
}

\author{
WangDongyu \\ Research Center of Intelligent Transportation System \\ Sun Yat-sen University
}

\author{
CaiMing* \\ Research Center of Intelligent Transportation System \\ Sun Yat-sen University \\ Guangzhou, China \\ caiming@mail.sysu.edu.cn \\ * Corresponding Author \\ Guangzhou, China
}

\begin{abstract}
The boundary element method (BEM) using collocation with constant elements has been adopted to calculate the insertion loss of a sound barrier in a twodimensional model. Analysis has been done on how the results obtained with BEM are affected by the numerical integration accuracy. First, the precision of all elements in matrix which were obtained through integration of equations with numerical integration approaches is directly influenced by the integration accuracy, which further affects the final results. Second, Two numerical integration methods, midpoint rectangle rule and composite four-point Gaussian quadrature rule, have been compared in this work. The number of integration points has relatively little effect on the results, and the composite four-point Gaussian rule has a higher convergence rate than the midpoint rectangle rule. Moreover,a comparison of wedge insertion loss calculated with GTD and BEM for different frequencies and different BEM element lengths has shown that: for wedge diffraction problems, it is necessary to ensure the element length is below 1/20 wavelength and consider the impacts of characteristic frequencies.
\end{abstract}

Keywords-Sound barrier; Diffraction; Boundary Element Method;The numerical integration accuracy.

\section{INTRODUCTION}

The spreading phenomenon of sound waves around obstacles is one important form of sound transmission. It is common in practical application to figure out the diffraction attenuation of sound waves from obstacles (such as sound barriers and buildings). After years of research, scholars have proposed lots of methods to calculate the attenuation of acoustic diffraction by obstacles, among which the empirical formula method [1], the Biot-Tolstoy method[2], the Hadden-Pierce method $[3,4]$ and the geometrical theory of diffraction (GTD) $[5,6]$ have been commonly used. As another common and effective numerical method, the boundary element method (BEM) has been nowadays widely used for this kind of problems with high calculation accuracy and excellent adaptability to complex boundaries. For instance, BEM has been used to determine the insertion loss of sound barriers of various shapes [7-10] and calculate the reactions to noise by the balcony of a building [11].

The computed results, which are induced via BEM through the Helmholtz equation for 2D sound fields, are affected the numerical integration accuracy. However, few literatures have explained in which way the BEM results of sound barrier diffraction problems are influenced by the numerical integration accuracy. To solve the problems of obstacle diffraction with BEM, it is essential to abide by specific requirements on boundary and accuracy. Therefore, this work was aimed at studying how the BEM results of sound barrier diffraction problems are affected by the numerical integration accuracy.To solve wedge diffraction problems, it is essential to abide by specific requirements on boundary and accuracy while dividing the element length (which is usually adopted at 1/4-1/20 wavelength by scholars for calculation [12-14]). Nevertheless, hardly any literature has compared the requirements on selecting element lengths for obstacles with different shapes especially with wedge. Therefore, this work was not only aimed at studying how the BEM results of sound barrier diffraction problems are affected by the numerical integration accuracy, but also comparing the requirements on element lengths for obstacles of wedge.

\section{THE BOUNDARY ELEMENT METHOD IN EQUATIONS FOR SOUND FIELDS}

\section{A. The establishment of the boundary integral equation}

As shown in Fig .1, suppose there is a two-dimensional sound field involving an infinite sound barrier with uniform cross section, and an acoustic line source which is parallel to both the sound barrier and the ground. $\mathrm{S}$ is taken as the boundary of the sound barrier, the acoustic source is 
located at $\mathrm{r} 0=(\mathrm{x} 0, \mathrm{y} 0)$, an arbitrary receiving point is located at $\mathrm{r}=(\mathrm{x}, \mathrm{y})$, thus in fluid medium, the sound pressure $\mathrm{p}(\mathrm{r}, \mathrm{r} 0)$ at the receiving point $\mathrm{r}$ satisfies the Helmholtz equation

$$
\left(\nabla^{2}+k^{2}\right) p\left(r, r_{0}\right)=\delta\left(r-r_{0}\right)
$$

$$
\text { Where } \nabla^{2}=\frac{\partial^{2}}{\partial x^{2}}+\frac{\partial^{2}}{\partial y^{2}} \text { is the Laplace operator; }
$$
$k=\frac{\omega}{c}$

$c$ is the wave number, $\omega$ is the wave frequency, $\mathrm{c}$ is the sound velocity; $\delta\left(r-r_{0}\right)$ represents the sound source at $\mathrm{r} 0$.

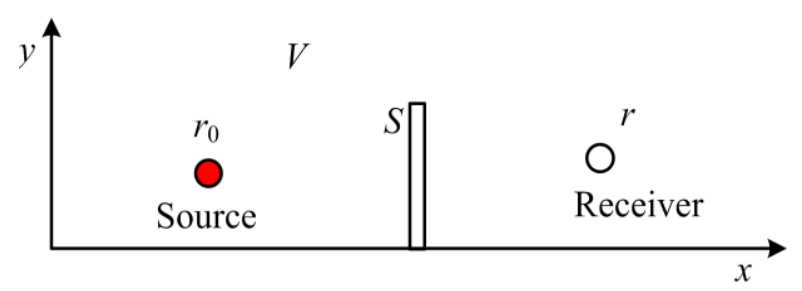

Figure 1. A two-dimensional sound field.

On the boundary, $\mathrm{p}(\mathrm{r}, \mathrm{r} 0)$ satisfies the impedance boundary conditions

$$
\frac{\partial p\left(r, r_{0}\right)}{\partial n}=i k \beta(r) p\left(r, r_{0}\right)
$$

Where $\mathrm{n}$ is the outward normal direction of the boundary; i stands for the imaginary unit; $\beta$ refers to the acoustic admittance of the boundary.

A boundary integral equation can be obtained according to Green's second integral formula, considering conditions of the boundary and properties of the $\delta$ function:

$$
\varepsilon(r) p\left(r, r_{0}\right)=G_{\beta c}\left(r, r_{0}\right)+\int_{S} p\left(r, r_{0}\right)\left(\frac{\partial G_{\beta c}\left(r_{s}, r\right)}{\partial n}-i k \beta\left(r_{s}\right) G_{\beta c}\left(r_{s}, r\right)\right) d S
$$

Where

$$
\varepsilon(r)= \begin{cases}1 & r \in V \\ \frac{1}{2} & r \in S \\ 0 & r \notin V \bigcup S\end{cases}
$$

Where $G \beta c(r, r 0)$ is a fundamental solution of the Helmholtz equation, suppose the ground is a fully rigid boundary, then $\mathrm{G} \beta \mathrm{c}(\mathrm{r}, \mathrm{r} 0)$ can be expressed as

$$
\begin{array}{r}
G_{\beta c}\left(r, r_{0}\right)=-\frac{i}{4}\left[H_{0}^{(1)}\left(k\left|r-r_{0}\right|\right)+H_{0}^{(1)}\left(k\left|r-r_{0}^{\prime}\right|\right)\right]_{(5)} \begin{array}{l}
\text { denote the sound pressure } \\
\text { then (3) can be written as }
\end{array} \\
\varepsilon(r) p\left(r, r_{0}\right)=G_{\beta c}\left(r, r_{0}\right)+\sum_{i=1}^{N} p\left(r_{i}, r_{0}\right) \int_{s_{i}}\left[\frac{\partial G_{\beta c}\left(r_{s_{i}}, r\right)}{\partial n}-i k \beta\left(r_{s_{i}}\right) G_{\beta c}\left(r_{s_{i}}, r\right)\right] \mathrm{d} s_{i}
\end{array}
$$
Hankel function of the first kind.

Here $r_{0}^{\prime}=\left(x_{0},-y_{0}\right)$ stands for the mirror image source of r0 relative to the ground; $H_{0}^{(1)}$ is a zero-order

\section{B. The boundary element method used to solve boundary integral equations}

Divide the boundary $\mathrm{S}$ discretely into $\mathrm{N}$ elements and mark them s1, s2,..sN. Suppose the elements are constant, assign the midpoints of such elements as nodes, and let the sound pressure level $\mathrm{p}(\mathrm{ri}, \mathrm{r} 0)$ at the midpoint of element $\mathrm{i}$ denote the sound pressure at any point within the element,

In the equation (6), let $r$ take on the values of $r 1$, $\mathrm{r} 2, \ldots, \mathrm{rN}$, then a group of $\mathrm{n}$-dimensional complex coefficient linear equations could be obtained, with respect to unknowns of $\mathrm{p}(\mathrm{r} 1, \mathrm{r} 0), \mathrm{p}(\mathrm{r} 2, \mathrm{r} 0), \ldots, \mathrm{p}(\mathrm{rN}, \mathrm{r} 0)$

$$
[A][P]=[G]
$$

All elements in matrix $[\mathrm{A}]$, vector $[\mathrm{P}]$ and vector $[\mathrm{G}]$ are complex numbers. Solve the linear equations, and put the obtained boundary sound pressure $\mathrm{p}(\mathrm{r} 1, \mathrm{r} 0), \mathrm{p}(\mathrm{r} 2$, $\mathrm{r} 0), \ldots, \mathrm{p}(\mathrm{rN}, \mathrm{r} 0)$ into equation (6), then for an arbitrary point in area $\mathrm{V}$, its sound pressure $\mathrm{p}(\mathrm{r}, \mathrm{r} 0)$ can be calculated.

Then $\Delta \mathrm{L}$, the insertion loss of the sound barrier, is given by the following formula

$$
\Delta L=20 \lg \frac{\left|p_{0}\left(r, r_{0}\right)\right|}{\left|p\left(r, r_{0}\right)\right|}
$$

Where $\mathrm{p} 0(\mathrm{r}, \mathrm{r} 0)$ stands for the sound pressure at the receiving point if the sound barrier takes no effect

$$
p_{0}\left(r, r_{0}\right)=-\frac{i}{4}\left[H_{0}^{(1)}\left(k\left|r-r_{0}\right|\right)+H_{0}^{(1)}\left(k\left|r-r_{0}^{\prime}\right|\right)\right]
$$

\section{THE IMPACTS OF THE ACCURACY OF NUMERICAL INTEGRATION ON THE RESULTS}

During the establishment of linear equations (7), all elements in matrix [A] were obtained through integration of (9) with numerical integration approaches. The precision of all elements in matrix [A] is directly influenced by the integration accuracy, which further affects the final results.

Two numerical integration methods, midpoint rectangle rule and composite four-point Gaussian 
quadrature rule, have been compared in this work. Under the midpoint rectangular rule, an integrating interval is equally divided into $\mathrm{k}$ subintervals, and the function value at the midpoint of a subinterval is taken as the value of the sub-interval (totally $\mathrm{k}$ computing points are taken). Whereas under the composite four-point Gaussian quadrature rule, the integrating interval is also equally divided into $\mathrm{k}$ subintervals, and 4 computing points are taken within each sub-interval in a certain proportion and weighting ( $4 \mathrm{k}$ computing points are taken in all). The $\mathrm{m}$ was set to 26 . The midpoint rectangle rule and the composite four-point Gaussian rule were respectively used in integration, and other conditions were the same as those in the previous example. The relationship between the number of computing points (taken during the integration of each element) and the final results of $\Delta \mathrm{L}$ has been presented in Fig .2. It is clear that the number of integration points has relatively little effect on the results, and the composite four-point Gaussian rule has a higher convergence rate than the midpoint rectangle rule. During calculation with the four-point Gaussian rule, an accuracy of 8 digits could be reached as long as more than 20 computing points are taken in each element.

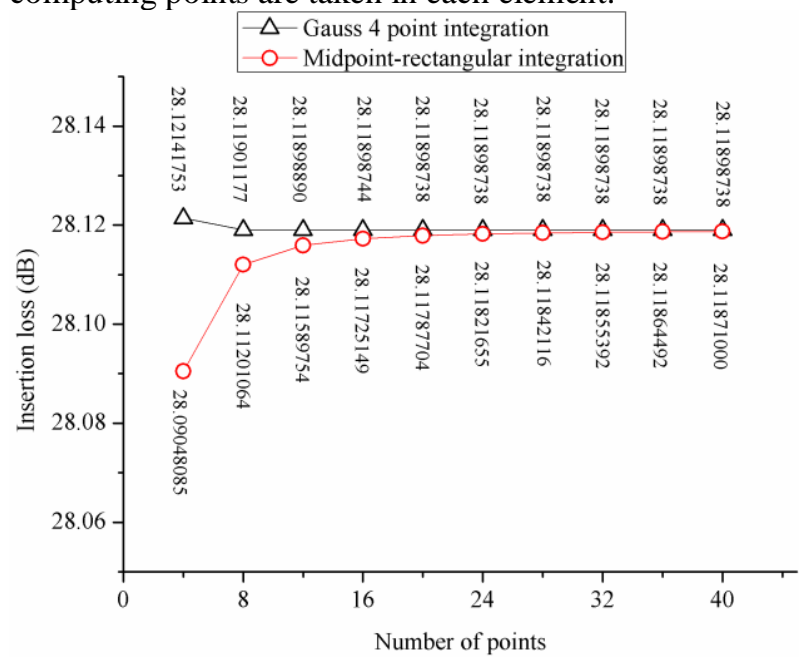

Figure 2. Relationship between the number of computing points and the insertion loss $\Delta \mathrm{L}$.

\section{ANALYSIS OF CALCULATION EXAMPLE OF WEDGE INSERTION LOSS}

The stability of BEM results will get worse if the sound barrier is changed over to a $90^{\circ}$ wedge of the same height, as shown in Fig 3. Even if the element length is shortened to $0.00707 \mathrm{~m}$, some deviation still arises above the high frequency of $3000 \mathrm{~Hz}(\lambda=0.113 \mathrm{~m})$. The results fit well with GTD results at any frequency below $3000 \mathrm{~Hz}$. However, unlike thin-screen and thick-screen problems, the wedge problems involve low characteristic frequencies of $340 \mathrm{~Hz}, 680 \mathrm{~Hz}, 1020 \mathrm{~Hz}, 1360 \mathrm{~Hz}$ and so on. The results deviate severely at any of the frequencies, since BEM yields more than one solutions at these special frequencies. This problem can usually be solved in two ways, one is the combined Helmholtz integral equation formulation (CHIEF) proposed by Schenck [15,16], the other is a method suggested by Burton and Miller to incorporate an equation for the normal derivative at the boundary [17]. The CHIEF method has been used herein to add integral equations for points that are out of the sound field (namely the case in equation (4), if $r \notin V \cup S$ ). Two points out of the field were taken, whose coordinates are $(0.1,2.0)$ and $(1.1,1.0)$. Thus, the equation (7) turns into over-determined equations, which are then solved with the least square method to get correct results. Above all, for the wedge diffraction problem, the results will not agree with GTD results unless the element length 1 is less than $\lambda / 20$. However, it is necessary to consider the influence of some characteristic frequencies.

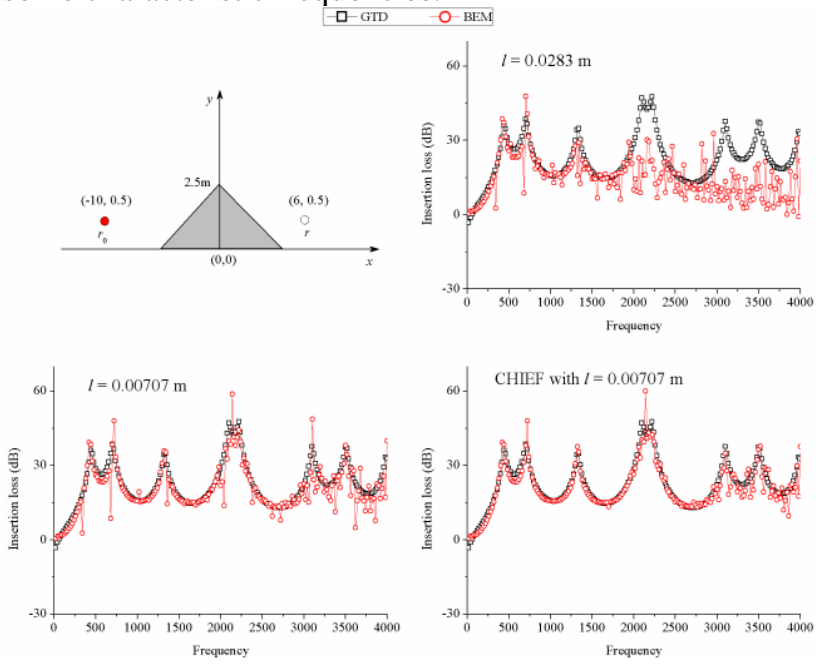

Figure 3. Comparison of wedge insertion loss calculated with GTD (squares) and BEM (circles) for different frequencies and different BEM element lengths.

\section{CONCLUSIONS}

In this work, the BEM has been used with constant elements to calculate two-dimensional sound diffraction problems of wedge. Then their results were compared with GTD results. Analysis has been done to find out how the results are influenced by the calculation accuracy of the numerical integration accuracy. Moreover, it has been discussed how to solve the problem that non-unique solutions arise from BEM at some characteristic frequencies.

The computed results are hardly affected by the numerical integration accuracy. However, the element division has relatively significant effects on such results. As to diffraction problems involving obstacles of wedge, the element must be shorter than $\lambda / 20$. Furthermore, it is necessary to avoid the impacts of characteristic frequencies during calculation.

\section{ACKNOWLEDGMENTS}

This work was supported by the Science and Technology Planning Project of Guangdong Province, China (No. 2015B010110005).

\section{REFERENCES}

[1] Z. Maekawa, Noise reducion by screens, Appl. Acoust. 1 (1968) 157-173.

[2] M. A. Biot and I. Tolstoy, Formulation of wave propagation in infinite media by normal coordinates with an application to diffraction, J. Acoust. Soc. Am. 29 (1957) 381-391. 
[3] W. T. Hadden and A. D. Pierce, Sound diffraction around screens and wedges for arbitrary point source locations, J. Acoust. Soc. Am. 69 (1981) 1266-1276.

[4] A. D. Pierce, Diffraction of sound around corners and over wide barriers, J. Acoust. Soc. Am. 55 (1974) 941-955.

[5] J. B. Keller, Geometrical Theory of Diffraction, J. Opt. Soc. Am. 52 (1962) 116-130.

[6] H. S. Kim, J. S. Kim, H. J. Kang, B. K. Kim and S. R. Kim, Sound diffraction by multiple wedges and thin screens, Appl. Acoust. 66 (2005) 1102-1119.

[7] T. Ishizuka and K. Fujiwara, Performance of noise barriers with various edge shapes and acoustical conditions, Appl. Acoust. 65 (2004) 125-141.

[8] K. Fujiwara, D. C. Hothersall and C. H. Kim, Noise barriers with reactive surfaces, Appl. Acoust. 53 (1998) 255-272.

[9] T. Okubo and K. Fujiwara, Efficiency of a sound barrier with an acoustically soft cylindrical edge for practical use, J. Acoust. Soc. Am. 105 (1999) 3326-3335.

[10] M. R. Monazzam and Y. W. Lam. Performance of profiled single noise barriers covered with quadratic residue diffusers, Appl. Acoust. 66 (2005) 709-730.
[11] D. C. Hothersall, K. V. Horoshenkov and S. E. Mercy, NUMERICAL MODELLING OF THE SOUND FIELD NEAR A TALL BUILDING WITH BALCONIES NEAR A ROAD, J. Sound Vib. 198 (1996) 507-515.

[12] A. T. Peplow, On the efficiency of screens near roadside cuttings, J. Sound Vib. 298 (2006) 982-1000.

[13] G. R. Watts and P. A. Morgan, Acoustic performance of an interference-type noise-barrier profile, Appl. Acoust. 49 (1996) 116.

[14] G. R. Watts, S. N. Chandler-Wilde and P. A. Morgan, The combined effects of porous asphalt surfacing and barriers on traffic noise, Appl. Acoust. 58 (1999) 351-377.

[15] H. A. Schenck, Improved integral formulation for acoustic radiation problems, J. Acoust. Soc. Am. 44 (1968) 41-58.

[16] W. Benthien and A. Schenck, Nonexistence and nonuniqueness problems associated with integral equation methods in acoustics, Comput. Struct. 65 (1997) 295-305.

[17] A. J. Burton and G. F. Miller, The Application of Integral Equation Methods to the Numerical Solution of Some Exterior BoundaryValue Problems, Proc. R. Soc. London. Ser. A, Math. Phys. Sci. 323 (1971) 201-210. 